\title{
A brief review on studies of Alzheimer's disease in China: Its mechanism, imaging and therapy
}

\author{
SHI Hua ${ }^{1,2} \&$ WANG Zhen ${ }^{1,2^{*}}$ \\ ${ }^{1}$ Britton Chance Center for Biomedical Photonics, Huazhong University of Science and Technology-Wuhan National Laboratory for \\ Optoelectronics, Wuhan 430074, China; \\ ${ }^{2}$ MOE Key Laboratory for Biomedical Photonics, Department of Biomedical Engineering, Huazhong University of Science and Technology, \\ Wuhan 430074, China
}

Received June 17, 2013; accepted July 8, 2013

Citation: Shi H, Wang Z. A brief review on studies of Alzheimer's disease in China: Its mechanism, imaging and therapy. Sci China Life Sci, 2013, 56: 1142-1144, doi: 10.1007/s11427-013-4566-1

\begin{abstract}
Alzheimer's disease (AD) was first described by German psychiatrist and neuropathologist Alois Alzheimer in 1906 and was named after him. It is the most common form of dementia and highly prevalent. AD is predicted to affect one in 85 people globally by 2050 . Well-known examples include Ronald Reagan, former US President, and Charles K. Kao, recipient of the 2009 Nobel Prize in Physics.

The neuropathology of $\mathrm{AD}$ is characterized by loss of neurons and synapses in cerebral cortex and certain subcortical regions. Both amyloid plaques and neurofibrillary tangles are clearly visible by microscopy in brains of those afflicted by AD. However, the cause for most Alzheimer's cases remains essentially unknown, except for $1 \%-5 \%$ of cases where genetic differences have been identified. Several competing hypotheses exist, trying to explain the cause of this disease, such as cholinergic hypothesis, amyloid hypothesis, and Tau hypothesis.
\end{abstract}

\section{Mechanism of AD}

Currently, China's population of old age has been increasing. $\mathrm{AD}$ is most often diagnosed in people over 65 years of age. AD becomes a prominent issue in old people, drawing more attention to studies on its mechanism, imaging for

*Corresponding author (email: zhenwang@mail.hust.edu.cn) diagnosis and treatment. In this article, we briefly review some progress made in this area by Chinese researchers.

Because $\mathrm{AD}$ is the most common cause of progressive cognitive decline in the aged population and most often diagnosed in people over 65 years of age, what is the relationship between brain aging and $\mathrm{AD}$ has to be considered. A relevant review was provided by Chui et al. This review addresses specific issues including whether $\mathrm{AD}$ and brain aging should be conceptually lumped or split, to what extent $\mathrm{AD}$ and brain aging potentially share common molecular mechanisms, whether $\beta$ amyloid $(A \beta)$ should be primarily considered as a marker of AD or simply that of brain aging, and what the definition of AD itself is. Moreover, an important common biochemical process shared by human aging and $\mathrm{AD}$, carbonyl stress, has been discussed by Yin et al. They investigated the upstream molecular causes of $\mathrm{AD}$, strategies based on this mechanism for treating $\mathrm{AD}$, as well as for preventing aging, fatigue and sub-health.

Amyloid hypothesis is prevalent for explaining the cause of $\mathrm{AD}$. Based on this hypothesis, the deposition of $\mathrm{A} \beta$ is a crucial progression in the pathophysiology of AD. It is also considered to be a promising candidate for AD therapy by decreasing its formation and/or increasing its clearance. In $\mathrm{A} \beta$ production cascade, there are some important enzymes, one of which is $A \beta$ precursor protein cleaving enzyme 1 (BACE1). By the regulation of BACE1, decreasing $\mathrm{A} \beta$ 
formation may be achieved. Therefore, Wang Tan and Sun XiuLian investigated the molecular regulation of BACE1 and its function at the early onset of AD. It was demonstrated that some of the pathological changes at the early onset of $\mathrm{AD}$, such as ischemia, hypoxia, inflammation and stress, are considered to be BACE1-related. Besides, it was suggested that many BACE1- and A $\beta$-related cytokines in fluids may become potential biomarkers, which would provide ideas for early clinical diagnosis of AD. Likewise, increasing its clearance is other potential way to treat $\mathrm{AD}$. Although $A \beta$ can be cleared through transportation to blood and cerebral spinal fluid pathway, most $A \beta$ peptide is degraded to small molecules by a protease called $\mathrm{A} \beta$ degrading enzyme such as neprilysin (NEP). A review on the role of NEP in the pathogenesis of AD was provided by Yang HongQi and Xing Ying in 2012. It was suggested that NEP may behave as a potential drug target for AD therapy.

Tau hypothesis is also considered to be a possible molecular mechanism underlying AD. Tau is the most abundant microtubule-associated protein. The normal function of Tau is to promote microtubule assembly and stabilize microtubules. In $\mathrm{AD}$, Tau is abnormally hyperphosphorylated and accumulated in the neuron to form neurofibrillary tangles. Wang JianZhi's group has systemically studied the mechanisms underlying Tau hyperphosphorylation and the effects of Tau phosphorylation on cell viability. It was proposed that Tau hyperphosphorylation may play a dual role in leading the neurons to abort from an acute apoptosis and at the same time trigger chronic neurodegeneration. It is implied that appropriate interference of Tau hyperphosphorylation may be used as a promising strategy in rescuing cell apoptosis and arresting neurodegeneration in AD. Furthermore, clinical trials indicated that some symptoms of $\mathrm{AD}$ patients can be alleviated by the supplementation of melatonin, which is an endogenously produced hormone in the brain and decreases in aged people and AD patients. The possible mechanism of melatonin function may be that it efficiently attenuates Tau hyperphosphorylation.

Except for the aforementioned competing hypotheses, recently there have been others trying to explain $\mathrm{AD}$. Among them, one hypothesis states that oxidative stress and dys-homeostasis of biometal (biology) metabolism may be important in the formation of AD pathology. The bio-metals of copper, zinc, iron, and manganese are examples that are essential for the normal functioning of the human body. Zhao BaoLu and Wan Li reviewed metal metabolic homeostasis disruption and early initiation of mechanism for AD. It was shown that the overload of iron and copper might have close relationships with oxidative stress damage in the later phase of $\mathrm{AD}$, while the deficiency of them might be relevant to the early initiation of AD. This may be useful for further research on the prevention and therapy of $\mathrm{AD}$.

There is also some latest development in studies of AD mechanism in China. Tong ZhiQian et al. performed an investigation and concluded that excess endogenous for- maldehyde induces memory decline. Scavenging excess formaldehyde may be a novel therapy for AD. What is more, $\mathrm{Su}$ Tao et al. showed effective assay brain endogenous formaldehyde. In addition, the decrease of brain estrogen was considered to be closely involved in female $\mathrm{AD}$ patients. Less estrogen receptor $\beta$ (ER $\beta$ ) was detected in mitochondrial, nuclear, and cytosolic fractions in female AD brains. Further studies of ER $\beta$ in mice suggested that ER $\beta$ might play roles in regulating mitochondrial function and affect the function of neurons accordingly.

\section{Imaging for AD}

Some imaging techniques have been used as clinical and diagnostic tools for $\mathrm{AD}$, and others are promising for $\mathrm{AD}$ studies. Here, we will briefly introduce them in terms of their imaging levels.

As an important instrument in neuroscience research, two-photon fluorescence light microscopy invented by Denk et al. improved three-dimensional in vivo imaging of cells and tissues. However, two-photon microscopy provided commercially can hardly meet the need for the detection of neural signal at millisecond scale due to its low imaging rates. Proposed by Zeng ShaoQun's group in China, fast random-access two-photon microscopy based on acoustooptic deflector has the potential to increase the observation speed while maintaining adequate signal-to-noise ratio. Jiang RunHua et al. from this group summarized the latest research progress. It was suggested that this technology may provide a new tool for neural activity observation and boost the development of brain science and it may be helpful for AD studies.

Some advanced medical imaging techniques have been available as diagnostic tools, such as computed tomography, magnetic resonance imaging, single-photon emission computed tomography, positron emission tomography, which can be synergistically used to confirm AD diagnosis. However, these diagnostic neuroimaging tools available in clinics have demonstrated only regional structural and functional changes instead of those in structural and functional networks in AD.

Recent progress in multi-modal imaging techniques and human brain connectome methods has allowed us to explore alterations of structural and functional networks in AD. More details about these techniques can be obtained in a review on advances in $\mathrm{AD}$ with neuroimaging presented by He Yong. Using these approaches, many studies have discovered AD-related network disruption, including connectivity strength, network efficiency, modular structure and network hubs. These studies provide novel insight into AD notion of network disintegration and might lead to the discovery of disease biomarkers for early diagnosis of AD.

In neuroscience, studies have been conducted concerning the brain-wide complex neural network of mammals' brains 
in physiological and pathological states. However, due to technological limitations, conventional imaging tools can only be used to study the local circuits of higher animals or the small brain network of lower animals. In 2010, Li AnAn et al. presented the whole brain imaging methods, which are promising at the level of optical microscopy and used to study the brain-wide complex neural network of mammals' brains. Furthermore, Li AnAn and Gong Hui reviewed recent progress in the methods at the level of optical microscopy.

\section{Treatments for AD}

It is well known that no treatment is available for stopping or reversing the progression of $\mathrm{AD}$. Current treatments are only helpful in attenuating the symptoms of AD. Based on the $A \beta$ cascade hypothesis, the inhibition of $A \beta$ production and aggregation as well as improvement of its clearance have been the main strategy for drug development. However, a disturbing issue is that no effective approach is available, although a large number of compounds have been in clinical trials. This issue probably can be explained by the following three reasons. Firstly, the $A \beta$ hypothesis that $A \beta$ is the key pathologic factor leading to $\mathrm{AD}$ progression might be questionable. Secondly, the loss of neural cells might be already too extensive, i.e., the poor clinical outcome of AD treatment is due to late intervention. New diagnostic ways may help to identify individuals before sub- stantial neural damage has occurred to them. Treatments could then be attempted to stave off the onset of the disorders or the progression of the disease. Thirdly, the failure to develop effective AD therapeutic agents in clinical trials could also be due to single-target or single-pathogenic pathway effect. The multi-target and multi-link treatment characteristics of traditional Chinese medicine have advantages over the single target treatment. The study of $\mathrm{Li}$ Lin and Zhang Lan showed that traditional Chinese medicine formula (Shenwu capsule) and single herb extracts (tetrahydroxy-stilbene glucoside, cornel iridoid glycoside, epimedium flavone and icariin) could act on the complex pathogenesis of $\mathrm{AD}$ at multi-targets and multi-pathways, especially having both neuroprotective and neurotrophic/ regenerative effects, and protect mitochondria and synapses, thus having significant features and advantages for the treatment of AD.

In brief, as the most common dementia in aged population, the cause for most $\mathrm{AD}$ remains unknown, as well as the mechanism of neuronal degeneration in AD. No treatment has been available for stopping or reversing the progression of $\mathrm{AD}$ until now. As China is stepping into 'aged society', AD becomes a prominent issue in old people, drawing increasing attention. The potential to answer questions on the cause and mechanisms of $\mathrm{AD}$ and to treat this devastating disease makes this time a critical one in $\mathrm{AD}$ research. As AD studies are flourishing in China, it is reasonable to think that further breakthroughs will be achieved.

Open Access This article is distributed under the terms of the Creative Commons Attribution License which permits any use, distribution, and reproduction in any medium, provided the original author(s) and source are credited. 